\title{
BALANCING, PELL AND SQUARE TRIANGULAR FUNCTIONS
}

\author{
AHMET TEKCAN, MERVE TAYAT, AND PÉTER OLAJOS
}

Received 22 June, 2015

\begin{abstract}
In this work, we derive some functions on balancing, cobalancing, Lucas-balancing, Lucas-cobalancing, Pell, Pell-Lucas and square triangular numbers. At the end of this article we investigated common values of combinatorial numbers and Lucas-balancing numbers.
\end{abstract}

2010 Mathematics Subject Classification: 11B37, 11B39, 11D25

Keywords: balancing numbers, Pell numbers, Pell-Lucas numbers, balancing functions, square triangular numbers

\section{INTRODUCTION}

A positive integer $n$ is called a balancing number (see [1] and [3]) if the Diophantine equation

$$
1+2+\cdots+(n-1)=(n+1)+(n+2)+\cdots+(n+r)
$$

holds for some positive integer $r$ which is called cobalancing number (or balancer). For example 6,35,204,1189 and 6930 are balancing numbers with balancers $2,14,84,492$ and 2870, respectively. If $n$ is a balancing number with balancer $r$, then from (1.1) one has $\frac{(n-1) n}{2}=r n+\frac{r(r+1)}{2}$, and so

$$
r=\frac{-(2 n+1)+\sqrt{8 n^{2}+1}}{2} \text { and } n=\frac{2 r+1+\sqrt{8 r^{2}+8 r+1}}{2} .
$$

Let $B_{n}$ denote the $n^{\text {th }}$ balancing number, and let $b_{n}$ denote the $n^{\text {th }}$ cobalancing number. Then they satisfy $B_{1}=1, B_{2}=6, B_{n+1}=6 B_{n}-B_{n-1}$ and $b_{1}=0, b_{2}=$ $2, b_{n+1}=6 b_{n}-b_{n-1}+2$ for $n \geq 2$. The zeros of the characteristic equation $x^{2}-$ $6 x+1=0$ for balancing numbers are $\alpha_{1}=3+\sqrt{8}$ and $\beta_{1}=3-\sqrt{8}$. Ray derived some nice results on balancing and cobalancing numbers in his Phd thesis [14, p.19]. Since $x$ is a balancing number if and only if $8 x^{2}+1$ is a perfect square, he set $y^{2}-8 x^{2}=1$ for some $y \neq 0$, which is a Pell equation. The fundamental solution is $\left(y_{1}, x_{1}\right)=(3,1)$. So $y_{n}+x_{n} \sqrt{8}=(3+\sqrt{8})^{n}$ and $y_{n}-x_{n} \sqrt{8}=(3-\sqrt{8})^{n}$ for $n \geq 1$.

This research was (partially) carried out in the framework of the Center of Excellence of Mechatronics and Logistics at the University of Miskolc. 
Thus $x_{n}=\frac{(3+\sqrt{8})^{n}-(3-\sqrt{8})^{n}}{2 \sqrt{8}}$ which is the Binet formula for balancing numbers and is denoted by $B_{n}$.

Let $\alpha=1+\sqrt{2}$ and $\beta=1-\sqrt{2}$ be the roots of the characteristic equation for Pell and Pell-Lucas numbers defined by $P_{0}=0, P_{1}=1, P_{n}=2 P_{n-1}+P_{n-2}$ and $Q_{0}=$ $Q_{1}=2, Q_{n}=2 Q_{n-1}+Q_{n-2}$ for $n \geq 2$, respectively. Notice that $\alpha^{2}=3+\sqrt{8}$ and $\beta^{2}=3-\sqrt{8}$. So the Binet formula for balancing numbers is $B_{n}=\frac{\alpha^{2 n}-\beta^{2 n}}{4 \sqrt{2}}$. Thus there is a connection between balancing and Pell numbers by $B_{n}=\frac{P_{2 n}}{2}$. Similarly, the Binet formula for cobalancing numbers is $b_{n}=\frac{\alpha^{2 n-1}-\beta^{2 n-1}}{4 \sqrt{2}}-\frac{1}{2}$, and so $b_{n}=$ $\frac{P_{2 n-1}-1}{2}$.

We note that $B_{n}$ is a balancing number if and only if $8 B_{n}^{2}+1$ is a perfect square, and $b_{n}$ is a cobalancing number if and only if $8 b_{n}^{2}+8 b_{n}+1$ is a perfect square. Thus (1.2) implies that

$$
C_{n}=\sqrt{8 B_{n}^{2}+1} \text { and } c_{n}=\sqrt{8 b_{n}^{2}+8 b_{n}+1}
$$

are integers called the $n^{\text {th }}$ Lucas-balancing and $n^{\text {th }}$ Lucas-cobalancing number, respectively. Their Binet formulas are $C_{n}=\frac{\alpha^{2 n}+\beta^{2 n}}{2}$ and $c_{n}=\frac{\alpha^{2 n-1}+\beta^{2 n-1}}{2}$, respectively (for further details see $[9,11-13]$ ).

Balancing numbers and their generalizations have been investigated by several authors, from many aspects. In [5, Theorem 4], Liptai proved that there is no Fibonacci balancing number except 1 , and in [6, Theorem 4] he proved that there is no Lucas balancing number. In [15], Szalay considered the same problem and obtained some nice results by a different method. In [4], Kovács, Liptai and Olajos extended the concept of balancing numbers to the $(a, b)$-balancing numbers defined as follows: Let $a>0$ and $b \geq 0$ be coprime integers. If

$$
(a+b)+\cdots+(a(n-1)+b)=(a(n+1)+b)+\cdots+(a(n+r)+b)
$$

for some positive integers $n$ and $r$, then $a n+b$ is an $(a, b)$-balancing number. The sequence of $(a, b)$-balancing numbers is denoted by $B_{m}^{(a, b)}$ for $m \geq 1$.

In [7], Liptai, Luca, Pintér and Szalay generalized the notion of balancing numbers to numbers defined as follows: Let $y, k, l \in \mathbb{Z}^{+}$such that $y \geq 4$. Then a positive integer $x$ with $x \leq y-2$ is called a $(k, l)$-power numerical center for $y$ if

$$
1^{k}+\cdots+(x-1)^{k}=(x+1)^{l}+\cdots+(y-1)^{l} .
$$

They studied the number of solutions of the equation above and proved several effective and ineffective finiteness results for $(k, l)$-power numerical centers.

For positive integers $k, x$, let

$$
\Pi_{k}(x)=x(x+1) \ldots(x+k-1) .
$$


It was proved in [4, Theorem 3 and Theorem 4] that the equation $B_{m}=\Pi_{k}(x)$ for fixed integer $k \geq 2$ has only infinitely many solutions and for $k \in\{2,3,4\}$ all solutions were determined. In [17, Theorem 1] Tengely, considered the case $k=5$, that is, $B_{m}=x(x+1)(x+2)(x+3)(x+4)$, and proved that this Diophantine equation has no solution for $m \geq 0$ and $x \in \mathbb{Z}$.

Recently in [2], Dash, Ota and Dash considered the so-called $t$-balancing numbers for an integer $t \geq 2$. A positive integer $n$ is a $t$-balancing number if

$$
1+2+\cdots+n=(n+1+t)+(n+2+t)+\cdots+(n+r+t)
$$

holds for some positive integer $r$ which is called $t$-cobalancing number. The $t$ balancing numbers are denoted by $B_{n}^{t}$ and $t$-cobalancing numbers by $b_{n}^{t}$. In [16], Tekcan, Tayat and Özbek derived the general terms of $t$-balancing numbers by solving the Diophantine equation $8 x^{2}-y^{2}+8 x(1+t)+(2 t+1)^{2}=0$.

Balancing functions are also interesting in the theory of balancing numbers. There were first considered by Behera, Panda and Ray in $[1,14]$, who derived the following results.

Lemma 1 ([1, Theorem 2.1]). For any balancing number $x$, the functions

$$
F(x)=2 x \sqrt{8 x^{2}+1}, G(x)=3 x+\sqrt{8 x^{2}+1} \text { and } H(x)=17 x+6 \sqrt{8 x^{2}+1}
$$

are also balancing numbers.

Lemma 2 ([1, Theorem 2.2]). If $x$ any balancing number, then

$$
K(x)=6 x \sqrt{8 x^{2}+1}+16 x^{2}+1
$$

is an odd balancing number.

Lemma 3 ([1, Theorem 4.1]). If $x$ and $y$ are balancing numbers, then

$$
f(x, y)=x \sqrt{8 y^{2}+1}+y \sqrt{8 x^{2}+1}
$$

is also a balancing number.

Lemma 4 ([14, 2.4.12 Theorem]). If $x, y$ and $z$ are balancing numbers, then

$$
\begin{aligned}
f(x, y, z)= & x \sqrt{8 y^{2}+1} \sqrt{8 z^{2}+1}+y \sqrt{8 x^{2}+1} \sqrt{8 z^{2}+1} \\
& +z \sqrt{8 x^{2}+1} \sqrt{8 y^{2}+1}+8 x y z
\end{aligned}
$$

is also a balancing number.

Similarly to balancing numbers, Panda and Ray [12] defined the following functions for any two cobalancing numbers $x$ and $y$ :

$$
\begin{aligned}
& f(x)=3 x+\sqrt{8 x^{2}+8 x+1}+1, \\
& g(x)=17 x+6 \sqrt{8 x^{2}+8 x+1}+8,
\end{aligned}
$$




$$
\begin{aligned}
h(x)= & 8 x^{2}+8 x+1+(2 x+1) \sqrt{8 x^{2}+8 x+1} \\
t(x, y)= & \frac{1}{2}\left[2(2 x+1)(2 y+1)+(2 x+1) \sqrt{8 y^{2}+8 y+1}\right. \\
& \left.+(2 y+1) \sqrt{8 x^{2}+8 x+1}+\sqrt{8 x^{2}+8 x+1} \sqrt{8 y^{2}+8 y+1}-1\right] .
\end{aligned}
$$

They proved the following two lemmas.

Lemma 5 ([12, Theorem 2.1]). For any two cobalancing numbers $x$ and $y, f(x)$, $g(x), h(x)$ and $t(x, y)$ are all cobalancing numbers.

Lemma 6 ([12, Theorem 2.2]). If $x$ any cobalancing number, then the cobalancing number next to $x$ is $f(x)=3 x+\sqrt{8 x^{2}+8 x+1}+1$, and consequently, the previous one is $\widetilde{f}(x)=3 x-\sqrt{8 x^{2}+8 x+1}+1$.

Here we note, [12] originally contained a type at $h(x)$, we always use the correct version.

\section{MAIN RESULTS}

In this section, we deal with balancing, Pell, Pell-Lucas and square triangular functions. Before considering balancing functions, we need the following theorem.

Theorem 1. For any integers $n, k, l \geq 0$, we get

$$
B_{n+k+l}=B_{k} C_{l} C_{n}+C_{k} B_{l} C_{n}+C_{k} C_{l} B_{n}+8 B_{k} B_{l} B_{n} .
$$

Proof. It is known that $B_{n+k}=C_{k} B_{n}+B_{k} C_{n}$ and $C_{k+l}=C_{k} C_{l}+8 B_{k} B_{l}$ for integers $n, l, k \geq 0$. So we easily get

$$
\begin{aligned}
& B_{k} C_{l} C_{n}+C_{k} B_{l} C_{n}+C_{k} C_{l} B_{n}+8 B_{k} B_{l} B_{n} \\
& =C_{n}\left(B_{k} C_{l}+C_{k} B_{l}\right)+B_{n}\left(C_{k} C_{l}+8 B_{k} B_{l}\right) \\
& =C_{n} B_{k+l}+B_{n} C_{k+l} \\
& =B_{n+k+l}
\end{aligned}
$$

as we wanted.

In Lemmas 1-5, Behara, Panda and Ray did not determine precisely value of the functions $F(x), G(x), H(x), K(x), f(x, y), f(x, y, z)$ and $f(x), g(x), h(x)$, $t(x, y)$. In the following theorem, we are able to do that.

Theorem 2. (1) For the balancing functions $F(x), G(x), H(x), K(x), f(x, y)$, $f(x, y, z)$, we have

$$
\begin{aligned}
& F\left(B_{n}\right)=B_{2 n}, G\left(B_{n}\right)=B_{n+1}, H\left(B_{n}\right)=B_{n+2}, K\left(B_{n}\right)=B_{2 n+1}, \\
& f\left(B_{n}, B_{k}\right)=B_{n+k} \text { and } f\left(B_{n}, B_{k}, B_{l}\right)=B_{n+k+l} .
\end{aligned}
$$


(2) For the cobalancing functions $f(x), g(x), h(x), t(x, y)$, we have $f\left(b_{n}\right)=b_{n+1}, g\left(b_{n}\right)=b_{n+2}, h\left(b_{n}\right)=b_{2 n}$ and $t\left(b_{n}, b_{m}\right)=b_{n+m}$.

Proof. (1) Since $\sqrt{8 B_{n}^{2}+1}=C_{n}$, we easily get

$$
F\left(B_{n}\right)=2\left(\frac{\alpha^{2 n}-\beta^{2 n}}{4 \sqrt{2}}\right)\left(\frac{\alpha^{2 n}+\beta^{2 n}}{2}\right)=\frac{\alpha^{4 n}-\beta^{4 n}}{4 \sqrt{2}}=B_{2 n} .
$$

Taking $k=1$ and $l=0$ in (2.1), one obtains

$$
B_{n+1}=3 B_{n}+C_{n}=3 B_{n}+\sqrt{8 B_{n}^{2}+1}=G\left(B_{n}\right) .
$$

If $k=2$ and $l=0$, then it leads to

$$
B_{n+2}=17 B_{n}+6 C_{n}=17 B_{n}+6 \sqrt{8 B_{n}^{2}+1}=H\left(B_{n}\right)
$$

and taking $k=1$ and $l=n$, we get

$$
B_{2 n+1}=6 B_{n} C_{n}+16 B_{n}^{2}+1=6 B_{n} \sqrt{8 B_{n}^{2}+1}+16 B_{n}^{2}+1=K\left(B_{n}\right) .
$$

Finally, if $l=0$, we have

$$
B_{n+k}=B_{n} C_{k}+B_{k} C_{n}=B_{n} \sqrt{8 B_{k}^{2}+1}+B_{k} \sqrt{8 B_{n}^{2}+1}=f\left(B_{n}, B_{k}\right) .
$$

The last assertions is obvious from (2.1) since

$$
\begin{aligned}
B_{n+k+l}= & B_{n} C_{k} C_{l}+B_{k} C_{n} C_{l}+B_{l} C_{n} C_{k}+8 B_{n} B_{k} B_{l} \\
= & B_{n} \sqrt{8 B_{k}^{2}+1} \sqrt{8 B_{l}^{2}+1}+B_{k} \sqrt{8 B_{n}^{2}+1} \sqrt{8 B_{l}^{2}+1} \\
& +B_{l} \sqrt{8 B_{n}^{2}+1} \sqrt{8 B_{k}^{2}+1}+8 B_{n} B_{k} B_{l} \\
= & f\left(B_{n}, B_{k}, B_{l}\right) .
\end{aligned}
$$

(2) It can be proved similarly.

Apart from above theorem, we can give the following theorem.

Theorem 3. (1) If $x$ is the $n^{\text {th }}$ balancing number and $y$ is the $n^{\text {th }}$ cobalancing number, then the function

$$
B_{1}(x, y)=\frac{-8 x y+\sqrt{8 x^{2}+1} \sqrt{8 y^{2}+8 y+1}+1}{4}
$$

is the $n^{\text {th }}$ balancing number, and the function

$$
B_{2}(x, y)=x+2 y+\sqrt{8 x^{2}+1}+\sqrt{8 y^{2}+8 y+1}+1
$$

is the $(n+1)^{\text {st }}$ balancing number. 
(2) (The generalized type of Lemma 2) Let $B_{k}$ denote the $k^{\text {th }}$ balancing number, let $b_{k}$ denote the $k^{\text {tk }}$ cobalancing number and let $C_{k}$ denote the $k^{\text {tk }}$ Lucasbalancing number for an integer $k \geq 0$. Then for any balancing number $x$, the function

$$
B_{k}^{1}(x)=B_{k} x \sqrt{8 x^{2}+1}+C_{k} x^{2}+1+2 b_{2 n+k}-\sum_{i=0}^{n-2} B_{2 i+k+1}
$$

is a balancing number. In fact if $x$ is the $n^{\text {th }}$ balancing number, then $B_{k}^{1}(x)$ is the $(2 n+k)^{\text {th }}$ balancing number. So $B_{k}^{1}(x)$ is odd balancing number for odd $k$ and even balancing number for even $k$.

(3) Let $B_{k}$ denote the $k^{\text {th }}$ balancing number, let $b_{k}$ denote the $k^{\text {tk }}$ cobalancing number and let $C_{k}$ denote the $k^{\text {th }}$ Lucas-balancing number for an integer $k \geq 0$. Then for any cobalancing number $x$, the function

$$
b_{k}^{1}(x)=C_{k} x+B_{k}\left(\sqrt{8 x^{2}+8 x+1}+1\right)+b_{k}
$$

is a cobalancing number. In fact, if $x$ is the $n^{\text {th }}$ cobalancing number, then $b_{k}(x)$ is the $(n+k)^{\text {th }}$ cobalancing number (Note that $b_{1}^{1}(x)=f(x)$ and $\left.b_{2}^{1}(x)=g(x)\right)$.

(4) If $x$ is the $n^{\text {th }}$ balancing number, $y$ is the $m^{\text {th }}$ cobalancing number and $z$ is the $n^{\text {th }}$ Lucas-balancing number, then the function

$$
b_{1}(x)=\frac{2 x+\sqrt{8 x^{2}+1}-1}{2}
$$

is the $(n+1)^{\text {th }}$ cobalancing number and the function

$$
b_{2}(x, y, z)=\frac{2 x \sqrt{8 y^{2}+8 y+1}+2 y \sqrt{8 x^{2}+1}+z-1}{2}
$$

is the $(n+m)^{\text {th }}$ cobalancing number.

Proof. (1) Notice that $B_{n}=\frac{\alpha^{2 n}-\beta^{2 n}}{4 \sqrt{2}}, b_{n}=\frac{\alpha^{2 n-1}-\beta^{2 n-1}}{4 \sqrt{2}}-\frac{1}{2}, C_{n}=\frac{\alpha^{2 n}+\beta^{2 n}}{2}$ and $c_{n}=\frac{\alpha^{2 n-1}+\beta^{2 n-1}}{2}$. So for any balancing number $x$ and cobalancing number $y$, we have

$$
\begin{aligned}
B_{1}(x, y) & =\frac{-8 x y+\sqrt{8 x^{2}+1} \sqrt{8 y^{2}+8 y+1}+1}{4} \\
& =\frac{-8\left(\frac{\alpha^{2 n}-\beta^{2 n}}{4 \sqrt{2}}\right)\left(\frac{\alpha^{2 n-1}-\beta^{2 n-1}}{4 \sqrt{2}}-\frac{1}{2}\right)+\left(\frac{\alpha^{2 n}+\beta^{2 n}}{2}\right)\left(\frac{\alpha^{2 n-1}+\beta^{2 n-1}}{2}\right)+1}{4} \\
& =\frac{\left\{\begin{array}{c}
\frac{-\alpha^{4 n-1}+\alpha^{2 n} \beta^{2 n-1}+\beta^{2 n} \alpha^{2 n-1}-\beta^{4 n-1}}{4}+\frac{\alpha^{2 n}-\beta^{2 n}}{\sqrt{2}} \\
+\frac{\alpha^{4 n-1}+\alpha^{2 n} \beta^{2 n-1}+\beta^{2 n} \alpha^{2 n-1}+\beta^{4 n-1}}{4}+1
\end{array}\right\}}{4}
\end{aligned}
$$




$$
\begin{aligned}
& =\frac{\frac{(\alpha \beta)^{2 n}\left(\alpha^{-1}+\beta^{-1}\right)}{2}+\frac{\alpha^{2 n}-\beta^{2 n}}{\sqrt{2}}+1}{4} \\
& =\frac{\alpha^{2 n}-\beta^{2 n}}{4 \sqrt{2}} \\
& =B_{n}
\end{aligned}
$$

since $\alpha \beta=-1$ and $\alpha^{-1}+\beta^{-1}=-2$. The others can be proved similarly.

As in Theorem 3, there are infinitely many Lucas-balancing and Lucas-cobalancing functions given below which can be proved similarly.

Theorem 4. Let $B_{k}$ denote the $k^{\text {th }}$ balancing number, $C_{k}$ denote the $k^{\text {th }}$ Lucasbalancing number and let $c_{k}$ denote the $k^{\text {tk }}$ Lucas-cobalancing number. Then for any balancing number $x$ and any cobalancing number $y$ with the same order, say $n$,

(1) the function

$$
C_{k}(x, y)=C_{k-1} \sqrt{8 x^{2}+1}+c_{k-1} \sqrt{8 y^{2}+8 y+1}+c_{n+k-1}
$$

is the $(n+k-1)^{\text {st }}$ Lucas-balancing number, that is, $C_{k}(x, y)=C_{n+k-1}$ for $k \geq 2$.

(2) the function

$$
c_{k}(x, y)=C_{k-1} \sqrt{8 y^{2}+8 y+1}+c_{k-1} \sqrt{8 x^{2}+1}+C_{n-k+1}
$$

is the $(n+k-1)^{s t}$ Lucas-cobalancing number, that is, $c_{k}(x, y)=c_{n+k-1}$ for $n \geq k-1 \geq 1$, and the function

$$
c_{k}(y)=4 B_{k}(2 y+1)+C_{k} \sqrt{8 y^{2}+8 y+1}
$$

is the $(n+k)^{t h}$ Lucas-cobalancing number, that is, $c_{k}(y)=c_{n+k}$.

For Pell and Pell-Lucas numbers, we can give the following theorem.

Theorem 5. Let $B_{k}$ denote the $k^{\text {th }}$ balancing number, let $b_{k}$ denote the $k^{\text {th }}$ cobalancing number, let $C_{k}$ denote the $k^{\text {tk }}$ Lucas-balancing number, let $c_{k}$ denote the $k^{\text {th }}$ Lucas-cobalancing number, let $P_{k}$ denote the $k^{\text {th }}$ Pell number and let $Q_{k}$ denote the $k^{\text {th }}$ Pell-Lucas number for an integer $k \geq 0$. Then for any balancing number $x$, for any cobalancing number $y$, for any Lucas-balancing number $z$ and for any Lucas-cobalancing number $w$ with the same order, say $n$,

(1) the function

$$
P_{k}^{1}(x, y)=\frac{8 B_{k}(x+y)+C_{k}\left(\sqrt{8 x^{2}+1}+\sqrt{8 y^{2}+8 y+1}\right)}{2}+P_{2 k}
$$


is the $(2 n+2 k)^{\text {th }}$ Pell number, that is, $P_{k}^{1}(x, y)=P_{2 n+2 k}$, the function

$$
P_{k}^{2}(x, y)=\frac{C_{k} \sqrt{8 x^{2}+1}+c_{k} \sqrt{8 y^{2}+8 y+1}}{2}
$$

is the $(2 n+2 k-1)^{\text {st }}$ Pell number, that is, $P_{k}^{2}(x, y)=P_{2 n+2 k-1}$, and the function

$$
P_{1}(x, y, z, w)=\frac{z \sqrt{8 x^{2}+1}+w \sqrt{8 y^{2}+8 y+1}}{2}
$$

is the $(4 n-1)^{\text {st }}$ Pell number, that is, $P_{1}(x, y, z, w)=P_{4 n-1}$.

(2) the function

$$
Q_{k}(x)=32 C_{k} x^{2}+32 B_{k} x \sqrt{8 x^{2}+1}+Q_{2 k}
$$

is the $(4 n+2 k)^{\text {th }}$ Pell-Lucas number, that is, $Q_{k}(x)=Q_{4 n+2 k}$.

(3) We set

$$
G_{k}(x, y)=B_{k} \sqrt{8 x^{2}+1}+b_{k} \sqrt{8 y^{2}+8 y+1} .
$$

(a) If $k=1$, then the function $G_{1}(x, y)$ is the $n^{\text {th }}$ Lucas-balancing number, that is, $G_{1}(x, y)=C_{n}$.

(b) If $k=2$, then the function $G_{2}(x, y)$ is the four times of the $(2 n+1)^{s t}$ Pell number, that is, $G_{2}(x, y)=4 P_{2 n+1}$.

(c) The function $G_{k}(x, y)$ is the sum of Lucas-balancing numbers from $n$ to $(n+k-1)$, that is,

$$
G_{k}(x, y)=\sum_{i=n}^{n+k-1} C_{i}
$$

for every $k \geq 1$, or is the sum of Pell numbers, that is,

$$
G_{k}(x, y)=\left\{\begin{array}{cc}
4 \sum_{i=1}^{\frac{k}{2}} P_{2 n+4 i-3} & \text { for even } k \geq 2 \\
P_{2 n}+P_{2 n-1}+4 \sum_{i=0}^{\frac{k-3}{2}} P_{2 n+4 i+3} & \text { for odd } k \geq 3 .
\end{array}\right.
$$

Proof. (1) Notice that $C_{n}+c_{n}=2 P_{2 n}, B_{n}+b_{n}=\frac{C_{n}-1}{2}$ and $P_{2 n}=2 B_{n}$. So

$$
\begin{aligned}
P_{k}^{1}(x, y) & =\frac{8 B_{k}(x+y)+C_{k}\left(\sqrt{8 x^{2}+1}+\sqrt{8 y^{2}+8 y+1}\right)}{2}+P_{2 k} \\
& =\frac{8 B_{k}\left(B_{n}+b_{n}\right)+C_{k}\left(C_{n}+c_{n}\right)}{2}+2 B_{k} \\
& =\frac{8 B_{k}\left(\frac{C_{n}-1}{2}\right)+2 C_{k} P_{2 n}}{2}+2 B_{k}
\end{aligned}
$$




$$
\begin{aligned}
& =2 B_{k} C_{n}+C_{k} P_{2 n} \\
& =2\left(\frac{\alpha^{2 k}-\beta^{2 k}}{4 \sqrt{2}}\right)\left(\frac{\alpha^{2 n}+\beta^{2 n}}{2}\right)+\left(\frac{\alpha^{2 k}+\beta^{2 k}}{2}\right)\left(\frac{\alpha^{2 n}-\beta^{2 n}}{2 \sqrt{2}}\right) \\
& =\frac{\left\{\begin{array}{c}
\alpha^{2(k+n)}+\alpha^{2 k} \beta^{2 n}-\beta^{2 k} \alpha^{2 n}-\beta^{2(n+k)} \\
+\alpha^{2(k+n)}-\alpha^{2 k} \beta^{2 n}+\beta^{2 k} \alpha^{2 n}-\beta^{2(k+n)}
\end{array}\right\}}{4 \sqrt{2}} \\
& =\frac{\alpha^{2(n+k)}-\beta^{2(n+k)}}{2 \sqrt{2}} \\
& =P_{2 n+2 k} .
\end{aligned}
$$

The others can be proved similarly.

There is a connection between balancing numbers and triangular numbers denoted by $T_{n}$ which are the numbers of the form $T_{n}=\frac{n(n+1)}{2}$ for $n \geq 0$. There are infinitely many triangular numbers that are also square numbers which are called square triangular numbers and is denoted by $S_{n}$. For the $n^{\text {th }}$ square triangular number $S_{n}$, we can write $S_{n}=s_{n}^{2}=\frac{t_{n}\left(t_{n}+1\right)}{2}$, where $s_{n}$ and $t_{n}$ are the sides of the corresponding square and triangle. Their Binet formulas are

$$
S_{n}=\left(\frac{\alpha^{2 n}-\beta^{2 n}}{4 \sqrt{2}}\right)^{2}, s_{n}=\frac{\alpha^{2 n}-\beta^{2 n}}{4 \sqrt{2}} \text { and } t_{n}=\frac{\alpha^{2 n}+\beta^{2 n}-2}{4} .
$$

In [10], Özkoç, Tekcan and Gözeri derived some nice results on triangular, square triangular and balancing numbers.

For the square triangular numbers, squares and triangles, we can give the following theorem.

Theorem 6. For any balancing number $x$ and any cobalancing number $y$ with the same order, say $n$,

(1) the function

$$
S(x, y)=\frac{4 x^{2}+4 y(y+1)+\sqrt{8 x^{2}+1} \sqrt{8 y^{2}+8 y+1}+1}{8}
$$

is the $n^{\text {th }}$ square triangular number, that is, $S(x, y)=S_{n}$.

(2) the function

$$
s(x, y)=\frac{6 y-2 \sqrt{8 x^{2}+1}+3 \sqrt{8 y^{2}+8 y+1}+3}{2}
$$

is the $(n-1)^{s t}$ square, that is, $s(x, y)=s_{n-1}$.

(3) the function

$$
t(x, y)=\frac{2(x-y-1)+\sqrt{8 x^{2}+1}-\sqrt{8 y^{2}+8 y+1}}{2}
$$


is the $n^{\text {th }}$ triangle, that is, $t(x, y)=t_{n}$.

Proof. (1) Since $1+\alpha^{-2}+2 \alpha^{-1}=1+\beta^{-2}+2 \beta^{-1}=2$ and $\alpha \beta=-1$, we get

$$
\begin{aligned}
S(x, y) & =\frac{4 x^{2}+4 y(y+1)+\sqrt{8 x^{2}+1} \sqrt{8 y^{2}+8 y+1}+1}{8} \\
& =\frac{\left\{\begin{array}{c}
4\left(\frac{\alpha^{2 n}-\beta^{2 n}}{4 \sqrt{2}}\right)^{2}+4\left(\frac{\alpha^{2 n-1}-\beta^{2 n-1}}{4 \sqrt{2}}-\frac{1}{2}\right)\left[\frac{\alpha^{2 n-1}-\beta^{2 n-1}}{4 \sqrt{2}}-\frac{1}{2}+1\right] \\
+\left(\frac{\alpha^{2 n}+\beta^{2 n}}{2}\right)\left(\frac{\alpha^{2 n-1}+\beta^{2 n-1}}{2}\right)+1
\end{array}\right\}}{8} \\
& =\frac{\alpha^{4 n}\left(1+\alpha^{-2}+2 \alpha^{-1}\right)+\beta^{4 n}\left(1+\beta^{-2}+2 \beta^{-1}\right)-4}{64} \\
& =\frac{\alpha^{4 n}-2(\alpha \beta)^{2 n}+\beta^{4 n}}{32} \\
& =\left(\frac{\alpha^{2 n}-\beta^{2 n}}{4 \sqrt{2}}\right)^{2} \\
& =S_{n} .
\end{aligned}
$$

The others can be proved similarly.

\section{NUMERICAL RESUlTS}

In [8], the authors dealt with the common values of balancing-type sequences. At the end of this paper we investigate this problem between Lucas-balancing and combinatorial numbers.

Let us consider the equation

$$
C_{n}=f(x) \text {, }
$$

where $f(x)$ is a polynomial with integer coefficients and $C_{n}$ is the $n^{\text {th }}$ Lucasbalancing number.

In this paper we study (3.1) when $f(x)$ is one of the polynomials $\left(\begin{array}{l}x \\ k\end{array}\right)$, and

$$
\begin{gathered}
S_{k}(x)=1^{k}+2^{k}+\cdots+(x-1)^{k}, \\
\Pi_{k}(x)=x(x+1) \ldots(x+k-1)
\end{gathered}
$$

for fixed integer $k \geq 2$.

We have to mention that we can deduce the following property of Lucas-balancing from equation (1.3):

Lemma 7. If $x= \pm C_{n}$ and $y= \pm B_{n}$, then the equation

$$
x^{2}-8 y^{2}=1
$$

is valid.

Our numerical result is the following. 
Theorem 7. Let $2 \leq k \leq 4$ and $f(x)$ be one of the polynomials $\left(\begin{array}{l}x \\ k\end{array}\right), \Pi_{k}(x)$, $S_{k-1}(x)$. Then the solutions $\left(C_{n}, x\right)$ of equation (3.1) are in the table

\begin{tabular}{|c|c||c|c|c|}
\hline$C_{m}$ & $B_{m}$ & $f(x)$ & $x$ & $k$ \\
\hline 3 & 1 & $\left(\begin{array}{l}x \\
k\end{array}\right)$ & 3 & 2 \\
\hline 3 & 1 & $S_{k-1}(x)$ & 3 & 2 \\
\hline
\end{tabular}

Proof. Consider the equation (3.1) when $f(x)$ one of polynomials $\left(\begin{array}{l}x \\ 2\end{array}\right),\left(\begin{array}{l}x \\ 3\end{array}\right)$ and $\left(\begin{array}{l}x \\ 4\end{array}\right)$. Using the multiplications by suitable powers of 2 and transformations $X=x$, $X=2(x-1)^{2}+1, X=x^{2}-3 x+1$ respectively, for the polynomials above. Then by Lemma 7 we obtain

$$
\begin{aligned}
\left(2^{3} y\right)^{2} & =2 X^{4}-4 X^{3}+2 X^{2}-8, \\
\left(2^{4} 3 y\right)^{2} & =X^{3}-7 X^{2}+15 X-297, \\
\left(2^{5} 3 y\right)^{2} & =2 X^{4}-4 X^{2}-1150,
\end{aligned}
$$

where $y$ is a balancing number. These types of equations are solvable by MAGMA (IntegralQuarticPoints and IntegralPoints), so after testing them we get the solutions above.

The suitable command at the first case is

IntegralQuarticPoints $([2,-4,2,0,-8],[3,8])$, because the point $[3,8]$ is on the curve. We get the solutions $(2,8),(2,0),(1,0),(3,8)$ for $\left(X, 2^{3} y\right)$. Using these results we realize that there is only one solution for $B_{n}, C_{n}$ and $x$, where $\left(B_{n}, C_{n}, x\right)=(1,3,3)$

Let us consider the second equation. In this case there is no solution, since from the property (1.3) follows that all Lucas-balancing numbers are odd, while $\left(\begin{array}{l}x \\ 3\end{array}\right)$ is even.

We use in the third case the command

IntegralQuarticPoints $([2,0,-4,0,-1150])$ and we found no solution.

Remark 1. We mention that we can use IntegralQuarticPoints ( [ ] ) in the case also when the constant term is not square, but the odd powers are missing from the right hand side.

Now let $f(x)$ be equal to $S_{k-1}(x)$. If $k=2$, then $S_{1}(x)=\left(\begin{array}{l}x \\ 2\end{array}\right)$, and its unique solutions has already been determined.

Applying the transformations $X=2(2 x-1)^{2}, X=\left(\begin{array}{l}x \\ 2\end{array}\right)$, respectively, to the equation (3.1) when $f(x)=S_{2}(x)$ and $f(x)=S_{3}(x)$ we get

$$
\begin{aligned}
\left(2^{6} 3 y\right)^{2} & =X^{3}-4 X^{2}+4 X-4608, \\
\left(2^{2} y\right)^{2} & =2 X^{4}-2 .
\end{aligned}
$$

Using the commands IntegralPoints (EllipticCurve $([0,-4,4,0,-4608]))$ and IntegralQuarticPoints $([2,0,0,0,-2])$, it provides only the solution $(1,0)$, in the second case, that is there is no solution for Lucas-balancing number. 
Finally let $f(x)=\Pi_{2}(x), \Pi_{3}(x), \Pi_{4}(x)$ and by using the transformations $X=$ $2 x+1, X=2(x+1)^{2}+1$ and $X=x^{2}+3 x$ we arrive that

$$
\begin{aligned}
\left(2^{4} y\right)^{2} & =2 X^{4}-4 X^{2}-30, \\
\left(2^{3} y\right)^{2} & =X^{3}-7 X^{2}+15 X-17, \\
y^{2} & =2 X^{4}+8 X^{3}+8 X^{2}-2 .
\end{aligned}
$$

By MAGMA, there exist no solution in the first and the second cases. In the third case we can easily conclude the same as follows. Since $X$ is always even, therefore $y$ is also even and we have

$$
y^{2} \equiv 0(\bmod 4) \text { and } 2 X^{4}+8 X^{3}+8 X^{2}-2 \equiv 2(\bmod 4),
$$

which is impossible.

\section{ACKNOWLEDGMENTS}

The authors wish to thank the anonymous referees for their detailed comments and suggestions which have improved the presentation of the paper.

\section{REFERENCES}

[1] A. Behera and G. K. Panda, "On the square roots of triangular numbers," Fibonacci Q., vol. 37, no. 2, pp. 98-105, 1999.

[2] K. K. Dash, R. S. Ota, and S. Dash, "t-balancing numbers," Int. J. Contemp. Math. Sci., vol. 7, no. 41-44, pp. 1999-2012, 2012.

[3] R. Finkelstein, “The house problem," Am. Math. Mon., vol. 72, pp. 1082-1088, 1965, doi: $10.2307 / 2315953$.

[4] T. Kovács, K. Liptai, and P. Olajos, “On $(a, b)$-balancing numbers,” Publ. Math., vol. 77, no. 3-4, pp. 485-498, 2010.

[5] K. Liptai, "Fibonacci balancing numbers," Fibonacci Q., vol. 42, no. 4, pp. 330-340, 2004.

[6] K. Liptai, "Lucas balancing numbers," Acta Math. Univ. Ostrav., vol. 14, no. 1, pp. 43-47, 2006.

[7] K. Liptai, F. Luca, A. Pintér, and L. Szalay, "Generalized balancing numbers," Indag. Math., New Ser., vol. 20, no. 1, pp. 87-100, 2009, doi: 10.1016/S0019-3577(09)80005-0.

[8] K. Liptai and P. Olajos, “About the equation $B_{m}^{(a, b)}=f(x)$," Ann. Math. Inform., vol. 40, pp. 47-55, 2012

[9] P. Olajos, "Properties of balancing, cobalancing and generalized balancing numbers," Ann. Math. Inform., vol. 37, pp. 125-138, 2010.

[10] A. Özkoç, A. Tekcan, and G. K. Gözeri, "Triangular and square triangular numbers involving generalized Pell numbers," Utilitas Mathematica, accepted.

[11] G. K. Panda, "Some fascinating properties of balancing numbers," Congr. Numerantium, vol. 194, pp. 185-189, 2009.

[12] G. K. Panda and P. K. Ray, "Cobalancing numbers and cobalancers," Int. J. Math. Math. Sci., vol. 2005, no. 8, pp. 1189-1200, 2005, doi: 10.1155/IJMMS.2005.1189.

[13] G. K. Panda and P. K. Ray, "Some links of balancing and cobalancing numbers with Pell and associated Pell numbers," Bull. Inst. Math., Acad. Sin. (N.S.), vol. 6, no. 1, pp. 41-72, 2011.

[14] P. K. Ray, "Balancing and cobalancing numbers," Ph.D. dissertation, Department of Mathematics, National Institute of Technology, Rourkela, India, 2009. 
[15] L. Szalay, "On the resolution of simultaneous Pell equations," Ann. Math. Inform., vol. 34, pp. 77-87, 2007.

[16] A. Tekcan, M. Tayat, and M. E. Özbek, "The Diophantine equation $8 x^{2}-y^{2}+8 x(1+t)+(2 t+$ $1)^{2}=0$ and $t$-balancing numbers," ISRN Comb., vol. 2014, p. 5, 2014, doi: 10.1155/2014/897834.

[17] S. Tengely, "Balancing numbers which are products of consecutive integers," Publ. Math., vol. 83, no. 1-2, pp. 197-205, 2013, doi: 10.5486/PMD.2013.5654.

Authors' addresses

Ahmet Tekcan

Uludag University, Faculty of Science, Department of Mathematics, Bursa-Turkiye

E-mail address: tekcan@uludag.edu.tr

\section{Merve Tayat}

Uludag University, Faculty of Science, Department of Mathematics, Bursa-Turkiye

E-mail address: mervetayat07@mail.com

Péter Olajos

University of Miskolc, Institute of Mathematics, Department of Applied Mathematics, Hungary

E-mail address: matolajeuni-miskolc.hu 